\title{
Comparison of cost-effectiveness of regorafenib and trifluridine/tipiracil combination tablet for treating advanced and recurrent colorectal cancer
}

\author{
MICHIO KIMURA ${ }^{1}$, EISEKI USAMI ${ }^{1}$, MINA IWAI ${ }^{1}$, MAKIKO GO $^{1}$, \\ HITOMI TERAMACHI $^{2}$ and TOMOAKI YOSHIMURA ${ }^{1}$ \\ ${ }^{1}$ Department of Pharmacy, Ogaki Municipal Hospital, Ogaki-shi, Gifu 503-8502; ${ }^{2}$ Laboratory of Clinical Pharmacy, \\ Gifu Pharmaceutical University, Gifu-shi, Gifu 501-1196, Japan
}

Received June 7, 2016; Accepted August 4, 2016

DOI: $10.3892 / \mathrm{mco} .2016 .1020$

\begin{abstract}
Regorafenib and trifluridine/tipiracil combination tablet regimens are standard third-line or later treatments for advanced and recurrent colorectal cancer with no significant difference in efficacy. The present study aimed to compare the cost-effectiveness of using regorafenib vs. the trifluridine/tipiracil combination tablet. The expected cost was calculated based on data from patients with advanced and recurrent colorectal cancer who were treated with regorafenib or trifluridine/tipiracil combination tablet. The median survival time (MST) from the CORRECT and the RECOURSE study was used to evaluate the therapeutic efficacy of the regimens. The cost-effectiveness ratio was calculated from the expected cost and MST for the two regimens. The expected cost per patient for the regorafenib and the trifluridine/tipiracil combination tablet regimen was $¥ 705,330.3$ and $¥ 371,198.7$, respectively, and the cost-effectiveness ratio was $¥ 110,207.9 / \mathrm{MST}$ and $¥ 52,281.5 / \mathrm{MST}$, respectively. In conclusion, the findings of the present study demonstrated that the trifluridine/tipiracil combination tablet regimen is more cost-effective compared with the regorafenib regimen.
\end{abstract}

\section{Introduction}

Combined chemotherapy regimens for colon cancer, including the combination of 5-fluorouracil (FU), leucovorin (LV) and oxaliplatin (FOLFOX), and the combination of capecitabine and oxaliplatin \pm bevacizumab, the combination of FU/LV and irinotecan (FOLFIRI) \pm cetuximab and FOLFOX \pm cetuximab,

Correspondence to: Dr Michio Kimura, Department of Pharmacy, Ogaki Municipal Hospital, 4-86 Minaminokawa-cho, Ogaki-shi, Gifu 503-8502, Japan

E-mail:kimkim0305nao@yahoo.co.jp

Key words: trifluridine/tipiracil combination tablet, regorafenib, cost-effectiveness, adverse event, advanced and recurrent colorectal cancer have assisted in prolonging survival (1-3). However, the high cost of these treatments has often been discussed (4).

The use of bevacizumab, cetuximab or panitumumab as second-line therapy for colon cancer after first-line chemotherapy is expensive. Therefore, it is important to apply the concept of drug economics for reducing medical expenses. The consideration of drug economics is more popular in western countries compared with in Japan. The effectiveness and economic efficiency of these treatments for advanced and recurrent colorectal cancer have been evaluated by numerous previous studies (5-15). For instance, for metastatic colorectal cancer, the incremental cost-effectiveness ratio (ICER) of XELOX as a first-line therapy was $£ 105,000$ per quality-adjusted life year (QALY) and that of first-line FOLFOX was $£ 108,000$ per QALY. The ICER of cetuximab for treating wild-type KRAS colorectal cancer was $¥ 16 \times 10^{6}$ per QALY (11). Therefore, none of these therapies is adequately cost-effective.

In general, the cost-effectiveness of anticancer agents for treating conditions with a short life expectancy (last line) is worsening. The American Society of Clinical Oncology and the National Comprehensive Cancer Centre Network recommend regorafenib or the trifluridine/tipiracil combination tablet as a third-line or later treatment for advanced and recurrent colorectal cancer. In Japan, this treatment is also recommended and is generally used (16). The trifluridine/tipiracil combination tablet is used based on the results of the RECOURSE study (17). In Europe, the Committee for Medicinal Products for Human Use of the European Medicines Agency has also approved this recommendation. For third-line or later treatment of advanced and recurrent colorectal cancer, no previous studies have, to the best of our knowledge, directly compared regorafenib and the trifluridine/tipiracil combination tablet.

The present study evaluated the cost-effectiveness of regorafenib vs. the trifluridine/tipiracil combination tablet as treatments for advanced and recurrent colorectal cancer in order to facilitate decision making during treatment selection.

\section{Patients and methods}

Treatment regimens. Regorafenib was administered at a dose of $160 \mathrm{mg} /$ day during 3-week courses with 1-week intervals 
Table I. Patient characteristics.

\begin{tabular}{|c|c|c|c|}
\hline Characteristic & Regorafenib & Trifluridine/tipiracil combination tablet & P-value \\
\hline Number & 10 & 34 & \\
\hline Median age, years (range) & $67.5(53-71)$ & $69(37-77)$ & 0.9491 \\
\hline No. males/females & $8 / 2$ & $18 / 16$ & 0.1211 \\
\hline ECOG performance status & & & 0.1211 \\
\hline 0 & 4 & 17 & \\
\hline 1 & 5 & 14 & \\
\hline 2 & 1 & 3 & \\
\hline Median number of previous treatment lines (range) & $3.5(3-5)$ & $3(3-5)$ & 0.1024 \\
\hline Median body surface area, $\mathrm{m}^{2}$, (range) & $1.69(1.34-1.82)$ & $1.53(1.09-1.96)$ & 0.2042 \\
\hline Disease status & & & 0.2175 \\
\hline Unresectable & 2 & 2 & \\
\hline Recurrent & 8 & 32 & \\
\hline Metastatic site & & & 0.4829 \\
\hline Liver & 6 & 19 & \\
\hline Lung & 3 & 15 & \\
\hline Peritoneum & 0 & 11 & \\
\hline Lymph node & 3 & 11 & \\
\hline Bone & 1 & 4 & \\
\hline Skin & 1 & 1 & \\
\hline
\end{tabular}

ECOG, Eastern Cooperative Oncology Group.

between the courses. The trifluridine/tipiracil combination tablet (with each dose consisting of $35 \mathrm{mg} / \mathrm{m}^{2}$ ) was administered twice daily after morning and evening meals for 5 days, followed by 2 days of rest and then again for 2 weeks, followed by a 14-day resting period, thus completing one treatment cycle. These are the patient data from the previous CORRECT and RECOURSE trials. The patients were administered two courses or more of either the regorafenib regimen $(n=10)$ or the trifluridine/tipiracil combination tablet regimen $(n=34)$ for treating advanced and recurrent colorectal cancer.

Calculation of cost. Cost data included direct costs occurring at the time of drug therapy. Fees for medication (including supportive care), inspection and medical examination of outpatients were calculated. Information on drug prices from the Insurance Drug Encyclopaedia (18) and medical fees from the Medical Fee Points Table (19) was retrieved to calculate total medical expenses. The cost of diagnostic imaging (chest computed tomography scan) and the labour of medical staff was included for each drug treatment and therefore did not require adding. The running and depreciation costs of facilities per patient were excluded as they were difficult to determine.

Determination of therapeutic efficacy. To obtain the therapeutic efficacy of the regorafenib and trifluridine/tipiracil combination tablet regimens, the CORRECT study (20) and the RECOURSE study (17) were used as data sources of the median survival time (MST).
Calculation of cost-effectiveness. The cost-effectiveness analysis was performed using the cost and effectiveness data of each drug regimen obtained as stated. The cost-effectiveness ratio of each drug regimen was calculated by dividing the expected cost by the MST. In addition, the ICER was determined to compare the cost-effectiveness of the trifluridine/tipiracil combination tablet regimen vs. that of the regorafenib regimen using the following equation: ICER $(¥ / \mathrm{MST})=($ expected cost of trifluridine/tipiracil combination tablet regimen - expected cost of regorafenib regimen) / (MST of trifluridine/tipiracil combination tablet regimen - MST of regorafenib regimen). Since the data available were for a time-period of no more than 1 year, the timing of the discount was not adjusted.

Analysis of adverse events (AEs). AEs were investigated for each patient retrospectively. The date of the occurrence of each AE was identified using electronic charts and pharmacy service records for the patients treated at Ogaki Municipal Hospital. The severity of AEs was classified according to the Common Terminology Criteria for Adverse Events (21).

Statistical analysis. Welch's t-test was used to analyse the variables and Student's t-test was used to analyse the number of outpatient visits and patient characteristics. In all significance tests, $\mathrm{P}<0.05$ was considered to indicate a statistically significant difference. All statistical analyses were performed using JMP 8 software (SAS Institute Inc., Cary, $\mathrm{NC}, \mathrm{USA})$ and the data are presented as the mean \pm standard deviation. 
Table II. Treatment costs.

\begin{tabular}{lcrr}
\hline Source of cost & Regorafenib & Trifluridine/tipiracil combination tablet & P-value \\
\hline Medication & & & $<0.0001$ \\
Anticancer drugs & $¥ 658,424.0$ & $¥ 349,685.0$ & 0.0184 \\
Supportive care drugs & $¥ 14,780.3$ & $¥ 5,535.0$ & $<0.0001$ \\
Inspection & $¥ 10,062.0$ & $¥ 3,453.5$ & $<0.0001$ \\
Outpatient medical examination & $¥ 6,716.0$ & $¥ 9,000.0$ & 1 \\
Management of malignant tumour-specific $_{\text {substances and therapeutic management }}$ & $¥ 9,000.0$ & & $<3,264.2$ \\
Others $^{\text {a }}$ & & $¥ 6,348.0$ & $<0001$ \\
Total & $¥ 705,330.3$ & $<0.0001$ \\
\hline
\end{tabular}

ancludes fees for providing drug information, prescriptions for outpatients, dispensing of oral preparations and basic fees for dispensing technology.

Table III. Cost-effectiveness ratio.

\begin{tabular}{lccc}
\hline Treatment & Expected cost per person & Cost-effectiveness ratio $^{\text {a }}$ & MST (months) $^{*}$ \\
\hline Regorafenib & $¥ 705,330.3$ & $¥ 110,207.9$ & 6.4 \\
Trifluridine/tipiracil combination tablet & $¥ 371,198.7$ & $¥ 52,281.5$ & 7.1 \\
P-value $^{\mathrm{b}}$ & $<0.0001$ & $<0.0001$ & NA \\
\hline
\end{tabular}

${ }^{a}$ The cost-effectiveness ratio was the expected cost per person/the effectiveness determined by the MST. ${ }^{\text {b }}$ egorafenib vs. trifluridine/tipiracil combination tablet. MST, median survival time; NA, not applicable.

Ethical considerations. The present study was approved by the Institutional Review Board of Ogaki Municipal Hospital (Ogaki, Japan).

\section{Results}

Patient characteristics. The patient characteristics are summarised in Table I. The median age of the patients who received regorafenib and trifluridine/tipiracil combination tablet regimens was 67.5 years (range, 53-71 years) and 69 years (range, 37-77 years), respectively, and the median number of previous treatments was 3.5 courses (range, 3-5 courses) and 3 courses (range, 3-5 courses), respectively.

Cost data. For the regorafenib regimen, the calculated direct medical cost included medication fee (anti-cancer drugs, $¥ 658,424.0$; supportive care drugs, $¥ 14,780.3$ ), an inspection fee of $¥ 10,062.0$ and an outpatient medical examination fee of $¥ 6,716.0$. For the trifluridine/tipiracil combination tablet regimen, the calculated direct medical cost included medication fee (anti-cancer drugs, ¥349,685.0; supportive care drugs, $¥ 261.0$ ), an inspection fee of $¥ 5,535.0$ and an outpatient fee of $¥ 3,453.5$. The regorafenib regimen was found to be more expensive compared with the trifluridine/tipiracil combination tablet regimen in total ( $¥ 705,330.3$ vs. $¥ 371,198.7 ; \mathrm{P}<0.0001$ ), as well as for each medical expense (Table II).
Cost-effectiveness analysis. The cost-effectiveness ratio (¥/month) was $¥ 110,207.9 / \mathrm{MST}$ for the regorafenib regimen and $¥ 52,281.5 / \mathrm{MST}$ for the trifluridine/tipiracil combination tablet regimen. A significant difference between the two groups was identified $(\mathrm{P}<0.0001$; Table III). The incremental cost-effectiveness ratio of the regorafenib regimen vs. the trifluridine/tipiracil combination tablet regimen was $¥ 477,330.9 / \mathrm{MST}$. The trifluridine/tipiracil combination tablet regimen was therefore found to be more cost-effective compared with the regorafenib regimen.

Number of outpatient visits. The number of outpatient visits in the first and second course was $4.6 \pm 0.5$ and $4.0 \pm 0.5$, respectively, for the regorafenib regimen, and 2.8 \pm 1.1 and $2.0 \pm 0.7$, respectively, for the trifluridine/tipiracil combination tablet regimen. Significant differences were observed between the two groups (Fig. 1).

AEs. The major AEs are summarised in Table IV. For the regorafenib regimen, these comprised hand-foot syndrome (70.0\%), malaise $(70.0 \%)$, hypertension $(60.0 \%)$, hoarseness $(60.0 \%)$ and an increase in aspartate transaminase and alanine transaminase $(50.0 \%)$. Among these AEs, $90.0 \%$ were grade 3 or higher; rhabdomyolysis was also observed. For the trifluridine/tipiracil combination tablet regime, major AEs comprised neutropenia $(61.8 \%)$, anaemia $(58.8 \%)$, nausea $(44.1 \%)$ and anorexia (20.6\%). Among these, $37.5 \%$ were grade 3 or higher. 
Table IV. Adverse events.

A, Patients treated with regorafenib $(n=10)$

\begin{tabular}{|c|c|c|c|c|c|}
\hline \multirow[b]{2}{*}{ Adverse event } & \multicolumn{4}{|c|}{ Grade } & \multirow[b]{2}{*}{ All grades, $\mathrm{n}(\%)$} \\
\hline & 1 & 2 & 3 & 4 & \\
\hline Thrombocytopenia & 1 & 0 & 1 & 0 & $2(20.0)$ \\
\hline Anaemia & 1 & 0 & 0 & 0 & $1(10.0)$ \\
\hline T-Bil increase & 0 & 1 & 2 & 0 & $3(30.0)$ \\
\hline AST/ALT increase & 0 & 2 & 2 & 1 & $5(50.0)$ \\
\hline CPK & 0 & 0 & 0 & 1 & $1(10.0)$ \\
\hline Malaise & 4 & 2 & 1 & 0 & $7(70.0)$ \\
\hline Anorexia & 2 & 0 & 1 & 0 & $3(30.0)$ \\
\hline Nausea & 2 & 0 & 0 & 0 & $2(20.0)$ \\
\hline Stomatitis & 2 & 0 & 0 & 0 & $2(20.0)$ \\
\hline HFS & 2 & 1 & 4 & 0 & $7(70.0)$ \\
\hline Rash (face) & 1 & 0 & 0 & 0 & $1(10.0)$ \\
\hline Hypertension & 2 & 1 & 3 & 0 & $6(60.0)$ \\
\hline Proteinuria & 0 & 1 & 0 & NA & $1(10.0)$ \\
\hline Hoarseness & 6 & 0 & 0 & 0 & $6(60.0)$ \\
\hline Fever & 2 & 0 & 0 & 0 & $2(20.0)$ \\
\hline
\end{tabular}

$\mathrm{B}$, Patients treated with trifluridine/tipiracil combination tablet $(\mathrm{n}=34)$

\begin{tabular}{lccccc}
\hline & \multicolumn{3}{c}{ Grade } & \\
\cline { 2 - 5 } Adverse event & 1 & 2 & 3 & 4 & All grades, n (\%) \\
\hline Neutropenia & 2 & 8 & 8 & 3 & $21(61.8)$ \\
Thrombocytopenia & 5 & 1 & 0 & 0 & $6(17.6)$ \\
Anaemia & 7 & 8 & 5 & 0 & $20(58.8)$ \\
T-Bil increase & 0 & 0 & 1 & 0 & $1(2.9)$ \\
AST/ALT increase & 0 & 1 & 0 & 0 & $1(2.9)$ \\
Creatinine increase & 2 & 0 & 0 & 0 & $2(5.9)$ \\
Malaise & 5 & 3 & 1 & 0 & $9(26.5)$ \\
Anorexia & 3 & 4 & 0 & 0 & $7(20.6)$ \\
Nausea & 12 & 0 & 3 & 0 & $3(44.1)$ \\
Vomiting & 3 & 0 & 0 & 0 & $1(2.9)$ \\
Stomatitis & 1 & 0 & 0 & 0 & $3(8.8)$ \\
Diarrhoea & 2 & 1 & 0 & 0 & $1(2.9)$ \\
Alopecia & 1 & 0 & NA & NA & $2(5.9)$ \\
Dysgeusia & 2 & 0 & NA & NA & $1(2.9)$ \\
Headache & 1 & 0 & 0 & 0 & $1(2.9)$ \\
Eye disorders (conjunctivitis) & 1 & 0 & 0 & 0 & $1(2.9)$ \\
Hyperkalaemia & 0 & 1 & 0 & 0 & \\
\hline
\end{tabular}

T-Bil, total bilirubin; HFS, hand-foot syndrome; AST, aspartate transaminase; ALT, alanine transaminase; CPK, creatine phosphokinase; NA, not determined.

\section{Discussion}

In the present study, a drug-economics analysis was performed to compare regorafenib and trifluridine/tipiracil combination tablet regimens as third-line or later treatments of advanced and recurrent colorectal cancer. It was revealed that the MST (months) was almost equal for the two regimens, and that the trifluridine/tipiracil combination tablet regimen has a superior cost-effectiveness compared with that of the regorafenib regimen. 


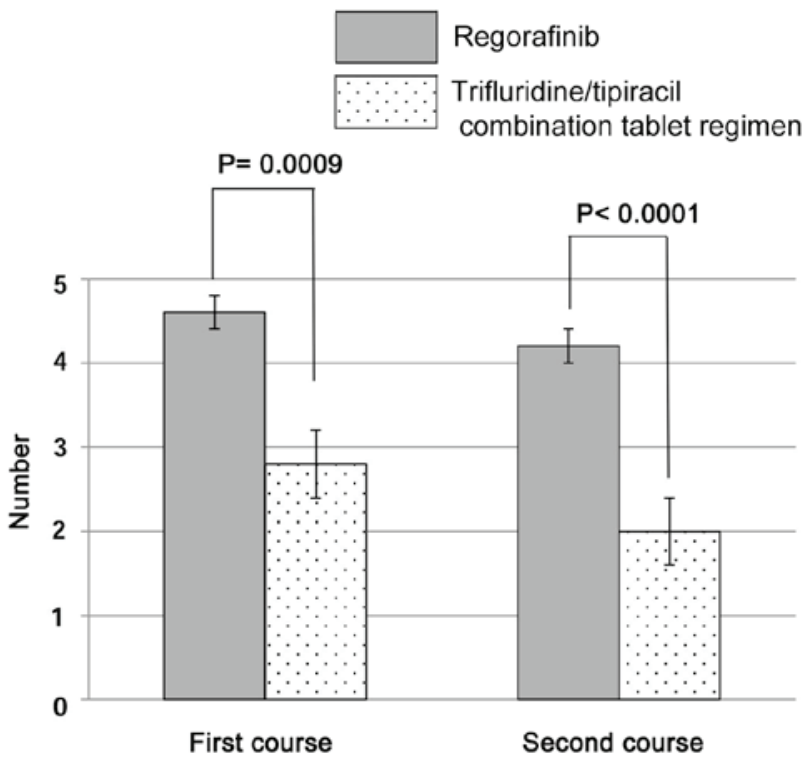

Figure 1. Number of outpatient visits during the courses of treatment. The data are presented as the mean \pm standard deviation.

The outcome (survival) is included in the cost-effectiveness value. Suppression of recurrence/progression of cancer, maintenance of quality of life (QOL) and extension of survival time are desired effects of anticancer agent treatments. However, advanced and recurrent cancer cannot be cured by these treatments. In certain cases, progression-free survival (PFS) and overall survival were extended from a few weeks to several months. The median PFS of patients with advanced and recurrent colorectal cancer treated with regorafenib and trifluridine/tipiracil combination tablet is only 1.9 and 2.0 months, respectively $(17,20)$.

Regarding cost-effectiveness, Goldstein et al (22) reported that regorafenib compared with the best supportive care, provides a minimal incremental benefit at high incremental cost per QALY in the third-line management of metastatic colorectal cancer. The present study found that the ICER for the trifluridine/tipiracil combination tablet regimen vs. regorafenib was $¥ 477,330.9 / \mathrm{MST}$. This figure objectively showed that patients can save almost $¥ 500,000$ /MST by opting for the trifluridine/tipiracil combination tablet regimen. However, the data on the cost determined by the present study are based on the patient population of a single facility. In the future, if data on cost are collected from several facilities, the results can be applied more widely.

Since the present study did not take QOL into account, it was not possible to accurately determine the cost-effectiveness in the common units $¥ /$ QALI. However, upon examining AEs, it was speculated that the high incidence of hand-foot syndrome (HFS) in patients subjected to the regorafenib regimen reduced their QOL. Go et al (23) reported that HFS and skin damage by anticancer agents decreased patient's QOL. In addition, patients receiving regorafenib more frequently presented with AEs of grade 3 or higher, including HFS, fatigue, high blood pressure and liver dysfunction. Thus, more frequent outpatient visits are required, which may represent a burden for patients. In the present study, the cost of supportive care agents for HFS and hypertension was added to the total cost of regorafenib.
As the cost of supportive care and outpatient visits was lower for patients receiving the trifluridine/tipiracil combination tablet regimen, it is considered an improved and more cost-effective treatment compared with regorafenib. The findings of the present study will assist medical practitioners and patients decide whether to use regorafenib or the trifluridine/tipiracil combination tablet for treating advanced and recurrent colorectal cancer.

In conclusion, the present study was the first to analyse the cost-effectiveness of two types of anticancer drug regimens for third-line or later treatment of advanced and recurrent colorectal cancer. The findings clearly suggested that the trifluridine/tipiracil combination tablet regimen is more cost-effective compared with regorafenib treatment.

\section{References}

1. Saltz LB, Clarke S, Díaz-Rubio E, Scheithauer W, Figer A, Wong R, Koski S, Lichinitser M, Yang TS, Rivera F, et al: Bevacizumab in combination with oxaliplatin-based chemotherapy as first-line therapy in metastatic colorectal cancer: A randomized phase III study. J Clin Oncol 26: 2013-2019, 2008.

2. Van Cutsem E, Köhne CH, Láng I, Folprecht G, Nowacki MP, Cascinu S, Shchepotin I, Maurel J, Cunningham D, Tejpar S, et al: Cetuximab plus irinotecan, fluorouracil, and leucovorin as first-line treatment for metastatic colorectal cancer: Updated analysis of overall survival according to tumor KRAS and BRAF mutation status. J Clin Oncol 29: 2011-2019, 2011.

3. Bokemeyer C, Bondarenko I, Hartmann JT, de Braud F, Schuch G, Zubel A, Celik I, Schlichting M and Koralewski P: Efficacy according to biomarker status of cetuximab plus FOLFOX-4 as first-line treatment for metastatic colorectal cancer: The OPUS study. Ann Oncol 22: 1535-1546, 2011.

4. Meropol NJ, Schrag D, Smith TJ, Mulvey TM, Langdon RM Jr, Blum D, Ubel PA and Schnipper LE; American Society of Clinical Oncology: American Society of Clinical Oncology guidance statement: The cost of cancer care. J Clin Oncol 27: 3868-3874, 2009.

5. Goldstein DA, Chen Q, Ayer T, Howard DH, Lipscomb J, El-Rayes BF and Flowers CR: First- and second-line bevacizumab in addition to chemotherapy for metastatic colorectal cancer: A United States-based cost-effectiveness analysis. J Clin Oncol 33: 1112-1118, 2015.

6. Carter HE, Zannino D, John Simes R, Schofield DJ, Howard K, Zalcberg JR, Price TJ and Tebbutt NC: The cost effectiveness of bevacizumab when added to capecitabine, with or without mitomycin- $\mathrm{C}$, in first line treatment of metastatic colorectal cancer: Results from the Australasian phase III MAX study. Eur J Cancer 50: 535-543, 2014.

7. Ruiz-Millo O, Albert-Mari A, Sendra-Garcia A and Jimenez-Torres NV: Comparative cost-effectiveness of bevacizumab-irinotecan-fluorouracil versus irinotecan-fluorouracil in first-line metastatic colorectal cancer. J Oncol Pharm Pract 20: 341-350, 2014.

8. Díaz-Rubio E, Pietrantonio F and de Braud F: Continuing single-agent bevacizumab as maintenance therapy after induction XELOX (or FOLFOX) plus bevacizumab in first-line treatment of metastatic colorectal cancer. Oncologist 17: 1426-1428, 2012.

9. Rinaldi F, George E and Adler AI: NICE guidance on cetuximab, bevacizumab, and panitumumab for treatment of metastatic colorectal cancer after first-line chemotherapy. Lancet Oncol 13: 233-234, 2012.

10. Tappenden P, Jones R, Paisley S and Carroll C: The cost-effectiveness of bevacizumab in the first-line treatment of metastatic colorectal cancer in England and Wales. Eur J Cancer 43: 2487-2494, 2007.

11. Shiroiwa T, Motoo Y and Tsutani K: Cost-effectiveness analysis of KRAS testing and cetuximab as last-line therapy for colorectal cancer. Mol Diagn Ther 14: 375-384, 2010.

12. Schwartzberg LS, Rivera F, Karthaus M, Fasola G, Canon JL, Hecht JR, Yu H, Oliner KS and Go WY: PEAK: A randomized, multicenter phase II study of panitumumab plus modified fluorouracil, leucovorin, and oxaliplatin (mFOLFOX6) or bevacizumab plus mFOLFOX6 in patients with previously untreated, unresectable, wild-type KRAS exon 2 metastatic colorectal cancer. J Clin Oncol 32: 2240-2247, 2014. 
13. Nomura H, Nagai S, Shinohara T, Iwashita M, Yajima M and Takatoya S: Compared medical costs of treating ovarian cancer patients with weekly paclitaxel, carboplatin (TC) chemotherapy. Gan To Kagaku Ryoho 34: 1091-1094, 2007 (In Japanese).

14. Inage S, Ise Y, Obayashi M, Katayama S and Gemma A: Cost-effectiveness analysis comparing carboplatin and weekly paclitaxel with cisplatin and docetaxel in the treatment of advanced non-small cell lung carcinoma. Gan To Kagaku Ryoho 37: 2093-2100, 2010 (In Japanese).

15. Kurihara T, Kobayashi M, Kogo M, Yoneyama K, Ito N, Sunaga T, Konishi K, Imawari M, Tobe T and Kiuchi Y: Cost-effectiveness analysis of chemotherapy with GEM or S-1 for patients with non-resectable pancreatic cancer. Gan To Kagaku Ryoho 37: 659-664, 2010 (In Japanese).

16. Watanabe T, Itabashi M, Shimada Y, Tanaka S, Ito Y, Ajioka Y, Hamaguchi T, Hyodo I, Igarashi M, Ishida $\mathrm{H}$, et al: Japanese Society for Cancer of the Colon and Rectum (JSCCR) guidelines 2014 for treatment of colorectal cancer. Int J Clin Oncol 20 207-239, 2015.

17. Mayer RJ, Van Cutsem E, Falcone A, Yoshino T, Garcia-Carbonero R, Mizunuma N, Yamazaki K, Shimada Y, Tabernero J, Komatsu Y, et al: Randomized trial of TAS-102 for refractory metastatic colorectal cancer. N Engl J Med 372: $1909-1919,2015$
18. Pharmaceutical Society: Insurance drug encyclopedia, Jiho, Tokyo, Pharmaceutical Society, 2012.

19. Kawakami Y: Medical fee points table. 35th edition. Social Insurance Institute, Tokyo, 2008.

20. Grothey A, Van Cutsem E, Sobrero A, Siena S, Falcone A, Ychou M, Humblet Y, Bouché O, Mineur L, Barone C, et al: Regorafenib monotherapy for previously treated metastatic colorectal cancer (CORRECT): An international, multicentre, randomised, placebo-controlled, phase 3 trial. Lancet 381: 303-312, 2013.

21. US Department Of Health And Human Services: Common terminology criteria for adverse events (CTCAE) version 4.0. United States, National Cancer Institute, 2009. (http://www. acrin.org/Portals/0/Administration/Regulatory/ CTCAE 4.02 2009-09-15 QuickReference 5X7.pdf).

22. Goldstein DA, Ahmad BB, Chen Q, Ayer T, Howard DH, Lipscomb J, El-Rayes BF and Flowers CR: Cost-effectiveness analysis of regorafenib for metastatic colorectal cancer. J Clin Oncol 33: 3727-3732, 2015.

23. Go M, Ikagawa M, Kimura M, Iwai M, Usami E, Yoshimura T and Yasuda K: Awareness of adverse events and health-related quality of life in outpatients receiving molecular targeted drugs for lung cancer patients. J Jpn Soc Hosp Pharm 51: 1462-1466, 2015 (In Japanese). 